NOTE

\title{
Copolymerization of Furan and Methyl $\alpha$-Cyanoacrylate
}

\author{
Eizo Orkawa, Fumio Aoki, and Kin-ichi Katano \\ Faculty of Engineering, Niigata University, Nagaoka 940, Japan.
}

(Received June 26, 1978)

KEY WORDS Furan / Methyl $\alpha$-Cyanoacrylate / Isoprene / NMR /

Furan is usually inactive with free-radical polymerization and the only comonomer that has been reported to copolymerize with furan is maleic anhydride, giving a 1:1 alternating copolymer. ${ }^{1}$ Butler, et al., ${ }^{1}$ reported that the structure of the resulting copolymer consisted of 2,5-addition of furan, while Kamo, et al., ${ }^{2}$ proposed the homopolymerization of Diels-Alder adduct of furan and maleic anhydride. However, Butler, et al., ${ }^{3}$ insisted on their structure giving additional evidence by quantitative determination of the degree of unsaturation in the polymer chain.

It has been suggested that electron-accepting monomers could effect the free-radical copolymerization with furan, ${ }^{1}$ but the report on such a subject has not yet appeared. We have attempted to prepare a copolymer of furan and methyl $\alpha$ cyanoacrylate, one of strong electron-accepting monomers, with a free-radical initiator, and obtain the copolymer. Its structure was studied by NMR and by the determination of the degree of unsaturation.

\section{EXPERIMENTAL}

\section{Materials}

Methyl $\alpha$-cyanoacrylate (MCA) was prepared from methyl cyanoacetate and paraformaldehyde, ${ }^{4}$ and stabilized by propanesultone. The copolymerizations were carried out in the presence of the stabilizer. A guaranteed grade of furan, purchased from Tokyo Kasei Kogyo Co., Ltd., was distilled over calcium hydride. Isoprene was treated in a similar manner.

\section{Copolymerization}

Furan, azobisisobutyronitrile (AIBN) and MCA were placed, in this order, with magnetic stirring, into a quartz tube filled with nitrogen gas, which had been placed in a water bath at room temperature. The mixture was irradiated with a $400-\mathrm{W}$ high-pressure mercury lamp. After the required time for such treatment, the mixture was poured into methanol. The precipitate was filtered and dried under reduced pressure. In the case of $\mathrm{CF}-5$ (Table I), a transparent gel was formed. It was separated from the liquid part by decantation, dissolved in nitromethane and poured in methanol to give a precipitate $(\mathrm{CF}-5 \mathrm{~b})$. It was reprecipitated from acetone by pouring into methanol. The liquid part was directly poured into methanol $(\mathrm{CF}-5 \mathrm{a})$. Both products showed a very similar IR pattern. A similar copolymerization procedure was followed in the isoprene-MCA copolymerization.

\section{Measurements}

The copolymer composition was analyzed with NMR spectra, using a JEOL Model JNMPMX 60 high-resolution spectrometer at $60 \mathrm{MHz}$ in DMSO- $d_{6}$ (for CF) and $\mathrm{CDCl}_{3}$ (for CIP) with tetramethyl silane as internal standard (Table I).

The iodine number was used for the determination of the degree of unsaturation for $\mathrm{CF}-5 \mathrm{~b}$. The method employed in this study was that adopted by Butler, et $a l^{3}{ }^{3}$ It is essentially the same as that applied by Rowe, et al., ${ }^{5}$ except for the use of acetic anhydride as solvent for the sample.

\section{RESULTS AND DISCUSSION}

The results of the copolymerization of furan with MCA are summarized in Table I and the copolymer composition against the monomer charge is shown in Figure 1. Since furan itself did not polymerize under similar conditions $(\mathrm{CF}-6)$, the products 


\section{E. Oikawa, F. Aoki, and K. Katano}

Table I. Copolymerization of furan (F) or isoprene (IP) with MCA at ambient temperature $\left(c a .25^{\circ} \mathrm{C}\right)^{\mathrm{a}}$

\begin{tabular}{|c|c|c|c|c|c|}
\hline No. & $\begin{array}{c}\text { Initial molar } \\
\text { ratio, } \\
\text { F or IP: MCA }\end{array}$ & $\begin{array}{l}\text { Irradiation } \\
\text { time, min }\end{array}$ & $\underset{\%}{\text { Conversion, }}{ }^{\mathrm{b}}$ & $\begin{array}{l}\text { Mole fraction } \\
\text { of } F \text { or IP } \\
\text { in product }\end{array}$ & {$[\eta]^{\mathrm{d}}$} \\
\hline $\mathrm{CF}-4$ & $3: 7$ & 105 & 46.2 & 0.27 & 0.67 \\
\hline $\mathrm{CF}-2$ & $5: 5$ & 60 & 39.1 & 0.39 & 0.36 \\
\hline $\mathrm{CF}-3$ & $7: 3$ & 150 & 34.0 & 0.41 & 0.49 \\
\hline CF-5 & 9:1 & 180 & $20.6^{\mathrm{e}}$ & 0.45 & 0.67 \\
\hline CF-6 & $10: 0$ & 120 & 0 & - & - \\
\hline $\mathrm{CIP}-2^{\mathrm{f}}$ & $5: 5$ & 180 & 46.1 & 0.52 & 1.34 \\
\hline CIP-9 ${ }^{\mathrm{f}}$ & $5: 5$ & 180 & 29.9 & 0.53 & 3.10 \\
\hline
\end{tabular}

a AIBN, $0.5 \mathrm{~mol} \%$ based on total monomers. No solvent was used in CF.

b Based on total monomer.

c Determined by NMR analysis.

d In DMF at $25^{\circ} \mathrm{C}$.

e CF-5a $0.9 \%, \mathrm{CF}-5 \mathrm{~b} 19.7 \%$. Determinations were made with $\mathrm{CF}-5 \mathrm{~b}$.

f IP concentration: $30 \mathrm{wt} \%$. Solvent: $\mathrm{CH}_{3} \mathrm{NO}_{2}$ for CIP-2 and $\mathrm{CH}_{3} \mathrm{CN}$ for CIP-9.

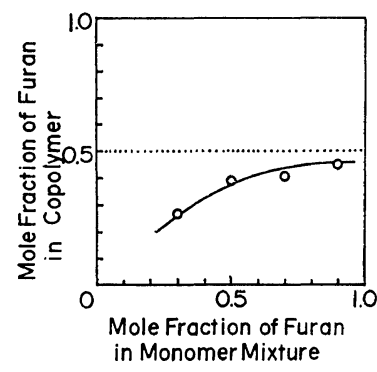

Figure 1. The copolymer composition curve in furan-MCA copolymerization.

must be copolymers of furan and MCA. This was also demonstrated by a solubility test; the $\mathrm{CF}$ products were soluble in acetone, acetonitrile, pyridine, dimethyl sulfoxide, $N$-methylpyrrolidone, and $N, N$-dimethylacetamide, whereas poly-MCA, prepared by bulk polymerization with AIBN $(0.5 \mathrm{~mol} \%)$ under ultraviolet irradiation, was partly soluble or swelled in the above solvents except acetone in which it was found to be completely insoluble.

It is of interest that the copolymer of furan and MCA was formed, since $p$-dioxane, 2-H-pyran, other unsaturated cyclic ether homologs, and isobutyl vinyl ether afforded no copolymers, but poly-MCA predominantly. These monomers, however, formed copolymers with vinylidene cyanide. ${ }^{6}$ In view of the facts that all the reagents used were carefully dried over calcium hydride, that no reaction occurred in $\mathrm{CF}-2$ without irradia- tion, and that 1:1-styrene-MCA copolymers were obtained in our experiment regardless of a wide range of monomer ratio $(8: 2-2: 8)$ as in a literature, ${ }^{7}$ it is evident that the anionic homopolymerization of MCA did not occur appreciably.

The structure of the copolymer was determined by the iodine number and NMR analysis. The duplicate test of the iodine number for $\mathrm{CF}-5 \mathrm{~b}$ gave 111 and 118 , but was calculated as 124.5 . The found values are lower than the calculated one by $5.2-11 \%$. This probably is due to the precipitation of some of the copolymer on addition of bromination reagent. The same phenomenon was observed in the furan-maleic anhydride copolymer. $^{3}$ The values of the iodine number rule out the possibility of homopolymerization of the Diels-Alder adduct. A possible structure is either I or II.
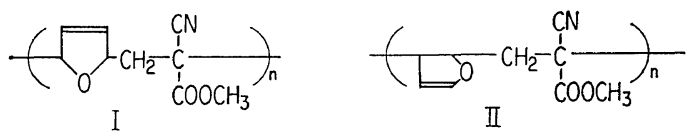

The NMR spectrum of CF-5b is shown in Figure 2. The peaks at $\delta 6.3$ and 3.1 may be assigned to $\mathrm{H}-\mathrm{C}=\mathrm{C}$ and $\mathrm{H}-\mathrm{C}-\mathrm{O}$, respectively, but these chemical bond shifts cannot determine the above structures, since 2, 3-dihydrofuran ${ }^{2}, 2$, 3-dihydrothiophene, ${ }^{8}$ maleic anhydride-thiophene copolymer $^{8}$ and maleic anhydride-furan adduct $(e x o)^{2}$ showed two peaks in the regions close to $\delta 6.3$ and 


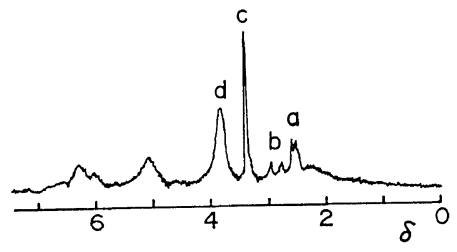

Figure 2. NMR spectrum of furan-MCA copolymer $(\mathrm{CF}-5 \mathrm{~b})$ in DMSO- $d_{6}$ at $60 \mathrm{MHz}$ : a, $\mathrm{CH}_{3}$ (DMSO); b, $\mathrm{CH}_{3}(\mathrm{DMF}) ; \mathrm{c}, \mathrm{H}_{2} \mathrm{O} ; \mathrm{d}, \mathrm{OCH}_{3}$.

5.1. In 2, 3-dihydrofuran and 2, 3-dihydrothiophene the peaks derived from $\mathrm{CH}_{2}$ were observed at $\delta 4.15$ and $2.47,{ }^{2}$ and at $\delta 3.2$ and $2.8,{ }^{8}$ respective1y. No such peaks are observed in Figure 2 except those occasioned by the presence of impurities. Moreover, the peak area ratio at $\delta 6.3,5.1$, and 3.9 is closely $2: 2: 3$. These facts suggest structure I. This is in agreement with the conclusion in the case of copolymerization of maleic anhydride with furan ${ }^{1,3}$ or with thiophene. ${ }^{8}$

The 2,5-addition of furan coincides with the structure of IP-MCA copolymers in Table I. Their single peaks at $\delta 5.27$ due to methyne $(=\mathrm{CH})$ and their patterns in NMR spectrum reveal that IP is present essentially 1, 4-trans configura- tion, since they are the same as found in the $1: 1$ IP-methyl methacrylate copolymer in which 1, 4trans IP was suggested. ${ }^{9}$ Furan and isoprene are common in conjugated diene structure. The conjugated bond of furan may interact with MCA, favoring the copolymerization despite the presence of oxygen.

\section{REFERENCES}

1. G. B. Butler, J. T. Badgett, and M. Sharabash, J. Macromol. Sci., Chem., A4, 51 (1970).

2. B. Kamo, J. Morita, S. Horie, and S. Furusawa, Polym. J., 6, 121 (1974).

3. Y. A. Ragab and G. B. Butler, J. Polym. Sci., Polym. Lett. Ed., 14, 273 (1976).

4. C. H. McKeever, U. S. Patent, 2, 912, 454 (1959).

5. R. G. Rowe, C. C. Furnas, and H. Bliss, Ind. Eng. Chem., Anal. Ed., 16, 371 (1944).

6. J. K. Stille and D. C. Chung, Macromolecules, 8, 114 (1975).

7. J. B. Kinsinger, J. R. Panchak, R. L. Kelso, J. S. Bartlett, and R. K. Grahem, J. Appl. Polym. Sci., 9, 429 (1965).

8. A. Cardon and E. J. Goethals, J. Macromol. Sci., Chem., A5, 1021 (1971).

9. E. Oikawa, A. Takagi, and T. Suzuki, Kobunshi Ronbunshu, 30, 22 (1973). 